A - Research concept and design

B - Collection and/or assembly of data

C - Data analysis and interpretation

D - Writing the article

E - Critical revision of the article

F - Final approval of article

Received: 2021-04-19

Accepted: 2021-07-19

Published: 2021-07-23

\section{Evaluation of tongue strength and symptoms of oral dysphagia in patients accompanying temporomandibular disorder}

\author{
Hazel Celik Guzel ${ }^{1, C-D}$ (D), Aysenur Tuncer*2,A-B,D-F (D) \\ ${ }^{1}$ Umut Rehabilitation Center Sanliurfa, Turkey \\ ${ }^{2}$ Faculty of Health Sciences, Department of Physiotherapy and Rehabilitation \\ Hasan Kalyoncu University Gaziantep, Turkey
}

*Correspondence: Aysenur Tuncer; Faculty of Health Sciences, Department of Physiotherapy and Rehabilitation, Hasan Kalyoncu University Gaziantep, Turkey; email: aysenur.tuncer@hku.edu.tr

\begin{abstract}
Introduction: The aim of this study was to compare functional restriction of the jaw, swallowing difficulty, tongue strength and endurance, and quality of life in temporomandibular disorder (TMD) patients with and without oral dysphagia (OD) and in healthy individuals.

Material and methods: The TMD group with 20 patients with TMD, and the TMD-OD group with 20 TMD patients with Eating Assessment Tool-10 (EAT-10) $\geq 3$, and presence of pain and difficulty in swallowing as well as control group comprising 20 healthy volunteers were included in this case-control study. Evaluations were made of pain severity, pain history, maximum mouth opening, Jaw Function Limitation Scale-20 (JFLS-20), and the Swallowing related-Quality of Life questionnaire (Swal-QoL). Additionally, the tongue strength and endurance were measured with the Iowa Oral Performance Instrument (IOPI).

Results: The history of pain, JFLS, EAT-10 values were significantly higher, and the Swal-QoL were significantly lower in the TMD-OD group compared to the patients with the TMD group $(\mathrm{p}<0.05)$. The IOPI values of the TMD and TMD-OD groups were determined to be significantly lower than those of the healthy group $(\mathrm{p}<0.05)$.

Conclusions: Functional restriction of the jaw, swallowing difficulty, and Swal-QoL showed a difference in the patients with TMD-OD compared to the patients with TMD only. The tongue strength and endurance values of the patients were different from those of the healthy control group.
\end{abstract}

Keywords: quality of life, temporomandibular joint disorders, swallowing disorders

\section{Introduction}

Temporomandibular disorders (TMD) is a disorder reported with irregularities related to the temporomandibular joint (TMJ) and associated musculoskeletal structures [1,2]. Pain and fatigue in the jaw muscles are the most frequently seen symptoms and accompanying symptoms may be seen of limitation of mouth opening, chewing difficulty, and head and neck pain [3].

The tongue plays a significant role in swallowing activity and provides normal swallowing function. Negative effects of the swallowing function as a consequence of impairment of the tongue are pathologies such as shaping, coordination, and opening movement

This is an Open Access journal, all articles are distributed under the terms of the Creative Commons Attribution-NonCommercial-ShareAlike 4.0 International (CC BY-NC-SA 4.0). License (http://creativecommons. org/licenses/by-nc-sa/4.0/). 
at the oral stage of swallowing. Adequate tongue muscle strength and endurance are determinant factors for safe and effective swallowing in the oral stage. Impairments in tongue function, such as limited strength, lack of co-ordination, or slow movement, can lead to several functional outcomes such as reduced tonguepalate pressure, weak bolus control, and less effective bolus transport, which can lead to oral dysphagia (OD) [4-6].

The prevalence of OD among TMD patients is $9.3 \%$ and is characterized by malpositioning of the tongue and over-contraction of the perioral and surrounding muscles [6,7]. Pain on chewing (87.4\%) and muscular fatigue (62\%) are common signs of OD among patients with TMD [7]. Hesitation and difficulty of swallowing, frequent repeated swallowing attempts, frequent throat clearance and cough associated with swallowing are symptoms of OD among TMD patients [8].

Instrumental evaluations are not required for oralinduced swallowing problems, but pharyngeal or laryngeal-induced swallowing problems as suggested by Logemann [9]. Videofluoroscopic (VFS) evaluation, which is the gold standard in evaluating swallowing disorders, has been used only once in TMD patients in the literature [10]. Besides, VFS requires special equipment, trained personnel and is expensive prevents it from being easily accessible. For this reason, studies have investigated the reliability of subjective dysphagia measurements using scales, such as EAT-10, by comparing them with objective data. It has been reported that the EAT-10 scale gives rapid and meaningful results in the evaluation of dysphagia and can be used in determining the risk of aspiration [11-13].

A few comparative studies investigated patients with OD problems accompanying TMD, by evaluation of correlation within the groups or with a healthy control group [14-17]. No study could be found in the literature that has investigated the differences between TMD patients with and without OD problems. Furthermore, there is no study that has shown the level of interaction between TMJ functionality in patients with TMD-OD and quality of life associated with OD. The hypothesis of this experimental study was that quality of life together with jaw functionality, dysphagia symptom severity, tongue strength, and endurance in TMD-OD patients would be different from those of TMD patients with no oral dysphagia. Therefore, the aim of this study was to compare functional restriction of the jaw, symptoms of oral dysphagia, tongue strength and endurance, and swallowing-related quality of life in TMD patients with and without oral dysphagia and in healthy individuals.

\section{Materials and methods}

The Human Research Ethics Committee approved the study protocol (decision no: 2019-131). All study procedures were in compliance with the principles of the World Medical Association Declaration of Helsinki. Written informed consent was obtained from all 60 participants.

Patients diagnosed as TMD by dental practitioners between October 2020 and February 2021 were included in this experimental study. Patients having at least 28 permanent teeth, aged between $18-65$ years old with the diagnosis of Group Ia, Ib, IIa, and III according to the Research Diagnostic Criteria for Temporomandibular Disorders (RDC/TMD) were included in the study [18]. Patients were excluded from the study if they had a diagnosis of Group II b,c according to the RDC/TMD [18] evaluation, a history of surgery regardless of TMJ problems in the cervical region, history of trauma, cancer, neurological problems, congenital anomalies, or musculoskeletal or systemic problems affecting the cervical region. In addition, patients who are edentulous or with no natural front teeth on which to get measuring points should be excluded.

The severity of pain in the jaw area was evaluated using the subjective measurement method of a $10 \mathrm{~cm}$ Visual Analog Scale (VAS) $(0=$ no pain, $10=$ intolerable pain) [19]. The maximum mouth opening was measured using a $15 \mathrm{~cm}$ ruler. The subjects were asked to open their mouth maximally till no further opening was possible. The distance from the incisal edge of the upper incisor teeth to the incisal edge of the lower incisor teeth was measured and the findings were recorded in ranges of millimeters. For reliability of the measurement and least error, correction of up to the amount of overbite was applied to the maximum opening movement value, and the average of 3 measurements was recorded [20]. The head posture of each subject was evaluated with the craniovertebral angle. It is the angle formed by the intersection of a horizontal line along the spinous process of $\mathrm{C} 7$ and the line drawn towards the ear tragus [21].

For the functionality evaluation, the Jaw Function Limitation Scale-20 (JFLS-20) was used. This reliable and valid scale, which was designed to measure the extent of restricted jaw function during different activities, consists of 20 items in the areas of chewing, vertical jaw movement, and verbal and emotional expression [22]. Each item is scored from $0-10$, evaluating the restriction related to that item, where 0 indicates no restriction and 10 indicates a severe restriction.

The diagnosis of oral dysphagia was made according to a score of 3 and above on the EAT-10 scale and the 
patient's self-report of pain and difficulty in swallowing. The Eating Assessment Tool-10 (EAT-10) was used for the evaluation of dysphagia symptoms and severity. EAT-10 is a validated, patient-reported, symptomspecific tool designed to rate self-perceived swallowing impairment. The scale comprises 10 items scored from 0 (no problem) to 4 (severe problem) to give a possible maximum score of 40 . Total points of $\geq 3$ indicate a swallowing problem [23].

Quality of life associated with swallowing problems was evaluated with the Swallowing Quality of Life Questionnaire (Swal-QoL). The Swal-QoL includes 10 concepts of general burden, food selection, eating duration, appetite, fear of food, sleep, fatigue, communication, mental health, and social function. High scores indicate good quality of life. The Swal-QoL is a valid and reliable test that has been used for the evaluation of the quality of life in previous studies related to swallowing disorders [24].

The tongue strength and endurance of the study subjects were evaluated with the Iowa Oral Performance Instrument (IOPI Medical LLC, Redmond, WA) [25]. Evaluation is made from the anterior and posterior of the tongue. The subjects are instructed to press the tongue on the tongue bulb towards the hard palate as hard as possible for 2 seconds. The maximum force (Pmax) was recorded as the highest value from 3 attempts. The tongue endurance measurements were calculated as $50 \%$ of the maximum tongue pressure previously measured, and the subjects were instructed to hold the tongue bulb with the tongue in the direction of the hard palate for as long as possible at this value. With the benefit of visual feedback, the subjects were instructed to keep the green light showing at the specified pressure only, and the duration for which it could be held was measured with a chronometer and recorded.

The groups were formed as Group 1 - TMD group $(\mathrm{n}=20)$, VAS score $\geq 4$; Group $2-$ TMD-OD group $(n=20)$, VAS score $\geq 4$, with EAT-10 scale points $\geq 3$, and presence of pain and difficulty in swallowing, and Group 3 - control group $(n=20)$, healthy individuals.

In calculating the power of the study, the effect size (0.60) obtained from the arithmetic mean and standard deviation values of anterior tongue strength in all three groups were used; the power was found to be 0.974 . The power of the study and the effect size of significant findings were calculated using G-Power 3.1.

\section{Statistical analysis}

Statistical analyses were performed by using MacOs-based SPSS vn. 26.0 software (SPSS Inc, Chicago,
IL, USA). Continuous variables were stated as mean \pm standard deviation (SD) values and categorical variables as number (n) and percentage (\%). Conformity of the data to normal distribution was assessed with the Kolmogorov-Smirnov test. In the comparison of tongue strength and endurance with maximum mouth opening, which has normally distributed, One-Way Anova analysis was used. Post-hoc Tukey test was applied for the grading of significant differences between the groups; The Independent t-test was used in the comparisons of pain and Swal-QoL parameters, normally distributed. Mann-Whitney U-test was used in the comparison of the JFLS-20 and EAT-10 findings which had not normal distribution. A value of $p<0.05$ was accepted as statistically significant.

\section{Results}

The mean age of the patients and control subjects was $29.40 \pm 5.36$ years in the TMD group, $32.15 \pm 7.78$ years in the TMD-OD group, and $29.55 \pm 8.46$ years in the control group. The three groups were homogenous in respect of age, gender, height, weight, body mass index (BMI), head posture, and marital status ( $p>0.05$ ). The demographic and clinical characteristics of the groups are presented in Table1.

No statistically significant difference was determined between the TMD and TMD-OD groups in respect of anterior and posterior tongue strength and tongue endurance values ( $p>0.05$ ). The tongue strength and endurance values of both the TMD and TMD-OD groups were determined to be statistically significantly lower than those of the healthy control group $(p<0.05)$. The values of maximum mouth opening were found to be statistically significant difference between the three groups, with the lowest value in the TMD-OD group $(\mathrm{p}<0.05)$ (Table 2).

There were no significant differences between the VAS values related to the TMJ between TMD and TMD-OD groups ( $>0.05$ ). The duration of complaints of pain was significantly longer in the TMD-OD group than in the TMD group $(\mathrm{p}<0.05)$ (Table 3$)$.

In the evaluation of the JFLS-20 values during different activities, jaw function restriction values were significantly higher in the TMD-OD group than in the TMD group $(p<0.05)$. Statistically significantly greater difficulty in swallowing was determined in the TMDOD patients $(\mathrm{p}<0.05)$ (Table 4).

All the subdimension values of the Swal-QoL demonstrating swallowing problem-related quality of life were statistically significantly lower in the TMD-OD group than in the TMD group ( $\mathrm{p}<0.05)$ (Tab. 5). 
Tab. 1. Demographic and clinical characteristic of the groups

\begin{tabular}{lcccc}
\hline & TMD $(\mathrm{n}=20)$ & TMD-OD $(\mathrm{n}=20)$ & Healthy $(\mathrm{n}=20)$ & $\mathrm{p}$ \\
\hline Age $($ years $)$ & $29.40 \pm 5.36$ & $32.15 \pm 7.78$ & $29.55 \pm 8.46$ & 0.416 \\
Height $(\mathrm{cm})$ & $168.85 \pm 7.65$ & $165.25 \pm 9.32$ & $171.45 \pm 7.16$ & 0.060 \\
Weight $(\mathrm{kg})$ & $66.25 \pm 13.81$ & $69.35 \pm 12.70$ & $68.40 \pm 13.91$ & 0.759 \\
BMI $\left(\mathrm{kg} / \mathrm{m}^{2}\right)$ & $23.08 \pm 3.67$ & $25.55 \pm 4.71$ & $23.16 \pm 3.94$ & 0.109 \\
Head Posture (degree) & $44.20 \pm 2.70$ & $43.90 \pm 4.24$ & $45.15 \pm 2.10$ & 0.558 \\
\hline $\mathrm{N}(\%)$ & & & \\
Sex & $12(60 \%)$ & $17(85 \%)$ & $15(75 \%)$ & 0.198 \\
Female & $8(40 \%)$ & $3(15 \%)$ & $5(25 \%)$ & \\
Male & & & \\
\hline $\mathrm{N}(\%)$ & & & \\
TMD classification & $9(45 \%)$ & $4(20 \%)$ & \\
Myofascial problems & $6(30 \%)$ & $9(45 \%)$ & \\
Disc problems & $5(25 \%)$ & $7(35 \%)$ & \\
Joint degeneration & & & \\
\hline
\end{tabular}

*p < 0.05; BMI: Body Mass Index; TMD:Temporomandibular Disorder; OD: Oral Dysphagia.

Tab. 2. Comparisons of tongue strength, endurance and maximum mouth opening values

\begin{tabular}{|c|c|c|c|c|c|c|}
\hline & $\begin{array}{c}\text { TMD } \\
\mathrm{X} \pm \mathrm{SD}(\mathrm{n}: 20) \\
\end{array}$ & $\begin{array}{c}\text { TMD-OD } \\
\mathrm{X} \pm \mathrm{SD}(\mathrm{n}: 20) \\
\end{array}$ & $\begin{array}{c}\text { Healthy } \\
\mathrm{X} \pm \mathrm{SD}(\mathrm{n}: 20)\end{array}$ & $\mathrm{F}$ & $\mathrm{p}$ & $\begin{array}{c}\text { Post hoc } \\
\text { comparisons }\end{array}$ \\
\hline Anterior Tongue Strength $(\mathrm{kPa})$ & $42.50 \pm 9.13$ & $40.60 \pm 8.43$ & $52.20 \pm 6.80$ & 11.56 & $0.000 *$ & $1,2-3$ \\
\hline Posterior Tongue Strength $(\mathrm{kPa})$ & $36.85 \pm 8.65$ & $35.30 \pm 8.87$ & $47.30 \pm 6.96$ & 12.64 & $0.000^{*}$ & $1,2-3$ \\
\hline Tongue Endurance (sn) & $19.70 \pm 4.73$ & $19.65 \pm 5.63$ & $26.70 \pm 6.75$ & 9.47 & $0.000 *$ & $1,2-3$ \\
\hline Maximum Mouth Opening (mm) & $38.15 \pm 5.14$ & $32.20 \pm 4.70$ & $43.00 \pm 3.09$ & 30.16 & $0.000^{*}$ & $1-2-3$ \\
\hline
\end{tabular}

*p < 0.05; p: One Way Anova, Post hoc comparisons: Tukey test; TMD: Temporomandibular Disorder; OD: Oral Dysphagia.

Tab. 3. Comparisons of pain intensity and pain history of the groups

\begin{tabular}{lcccc}
\hline Pain & TMD & TMD-OD & p & Effect size \\
& $\mathrm{X} \pm$ SD (n:20) & $\mathrm{X} \pm$ SD (n:20) & 0.178 & - \\
VAS $(0-10 \mathrm{~cm})$ & $4.75 \pm 0.91$ & $5.20 \pm 1.15$ & $0.000^{*}$ & 1.506 \\
Pain History (years) & $0.72 \pm 0.39$ & $3.31 \pm 2.41$ & \\
\hline
\end{tabular}

${ }^{*} \mathrm{p}<0.05$, p: Independent $\mathrm{t}$ test; TMD: Temporomandibular Disorder; OD: Oral Dysphagia.

Tab. 4. Comparisons of jaw function limitation and swallowing disorder severity values

\begin{tabular}{lcccc}
\hline & TMD & TMD-OD & p & Effect size \\
\hline JFLS-20 & $21.25 \pm 11.31$ & $47.90 \pm 16.85$ & $0.000^{*}$ & 1.850 \\
EAT-10 & $0.45 \pm 0.51$ & $12.20 \pm 5.29$ & $0.000^{*}$ & 3.126 \\
\hline
\end{tabular}

*p < 0.05; p: Mann Whitney U Test; JFLS-20: Jaw Function Limitation Scale; EAT-10: Eating Assessment Tool; TMD: Temporomandibular Disorder; OD: Oral Dysphagia. 
Tab. 5. Comparisons of the swallowing-related quality of life parameters

\begin{tabular}{lcccc}
\hline Swal-QoL & TMD & TMD-OD & \multirow{2}{*}{ Effect size } \\
\hline General burden & 100.00 & $81.87 \pm 11.08$ & $0.000^{*}$ & 2.313 \\
Food selection & 100.00 & $62.50 \pm 13.45$ & $0.000^{*}$ & 3.942 \\
Eating duration & $95.00 \pm 8.50$ & $73.12 \pm 4.57$ & $0.000^{*}$ & 3.206 \\
Eating desire & $97.00 \pm 5.34$ & $76.52 \pm 7.31$ & $0.000^{*}$ & 3.199 \\
Fear of eating & 100.00 & $76.00 \pm 10.43$ & $0.000^{*}$ & 3.254 \\
Sleep & $93.75 \pm 11.10$ & $70.62 \pm 20.38$ & $0.000^{*}$ & 1.409 \\
Fatigue & $98.75 \pm 5.59$ & $67.07 \pm 10.97$ & $0.000^{*}$ & 3.638 \\
Communication & 100.00 & $83.12 \pm 9.31$ & $0.000^{*}$ & 2.564 \\
Mental health & 100.00 & $70.00 \pm 12.77$ & $0.000^{*}$ & 3.322 \\
Social Functioning & 100.00 & $84.25 \pm 14.19$ & $0.000^{*}$ & 1.569 \\
Total Scale & $98.45 \pm 1.72$ & $75.38 \pm 8.78$ & $0.000^{*}$ & 3.646 \\
\hline
\end{tabular}

${ }^{*} \mathrm{p}<0.05$, p: Independent t-test, Swal-QoL: Swallowing-related Quality of life; TMD: Temporomandibular Disorder; OD: Oral Dysphagia.

\section{Discussion}

The aim of this study was to compare functional restriction of the jaw, swallowing difficulty, tongue strength and endurance, and swallowing-related quality of life in TMD patients with and without symptoms of oral dysphagia and in healthy individuals. The study hypothesis that there would be a difference in jaw functionality, dysphagia symptom severity, and swallowing-related quality of life between TMD-OD patients and TMD patients without swallowing problems was partially confirmed, with the exception of the tongue strength and endurance values. No difference was found in respect of tongue strength and endurance values in the group comparisons, but all the patients with TMD were seen to have significantly lower tongue strength and endurance, independently of swallowing problems, compared to the healthy control group.

Objective measurements of the strength and endurance of tongue muscles contribute to the clinical evaluation of dysphagia. Patients with OD have difficulty in transferring food from the mouth to the pharynx and esophagus to start the involuntary swallowing process. The development of TMD may be the cause of changes in joint movements, a decrease in the mandibular opening movement, and chewing impairment [26]. It has been hypothesized that eating and swallowing problems in patients with TMD develop secondary to chewing disorders [27]. It has also been reported that signs and symptoms of swallowing problems such as chewing pain, fatigue, swallowing difficulty, difficulty in fluid intake, and an increase in neck muscle activity are seen in approximately $10 \%$ of patients with TMD [7].

Previous studies have shown that $95 \%$ of the normal population, including the elderly, have a Pmax of tongue strength values $>34 \mathrm{kPa}$. Posterior tongue strength is generally $5-10 \%$ lower than anterior tongue strength. However, there has been reported to be insufficient data as yet for the norm distributions of tongue endurance $[28,29]$. Resistance of $\leq 10$ secs may be an indicator that the patient has low resistance and low endurance, and it has been reported that in such cases, fatigue is a factor in oral-motor problems [25,28-30]. There are studies in the literature showing a significant correlation between the maximum isometric pressure of the tongue and the mean swallowing pressures used during swallowing [30]. A systematic review that examined the results of isometric tongue strength training in patients with dysphagia showed that the tongue strength of dysphagia patients was lower than that of healthy individuals [31].

Some researchers have hypothesized that patients with chronic TMD have irregular or weak tongue functions [14-17]. In another study, it was shown that the genioglossus muscle, which is closely related to oral functions such as chewing and swallowing, moved in coordination with and as a functional connection to digastric muscles, which function in TMJ elevation [32]. In central pattern generators, genioglossus activity has been found to be associated with the activity of chewing muscles [33]. Fassicollo et al., compared patients with chronic TMD with a healthy control group and reported 
that in individuals with TMD, muscle activity during swallowing activity requires a longer time to reach peak values, and a longer swallowing time is needed. It was found that TMD patients contract the jaw elevator muscles more than the control group and the suprahyoid muscle peak values were significantly lower in fluid swallowing activity [17]. In the current study, we compared TMD patients with and without oral stage swallowing problems and with healthy subjects. The tongue strength and endurance values of all the TMD patients were seen to be significantly lower than those of the healthy control group. However, the difference in tongue strength expected in the current study hypothesis was not determined between the TMD and TMD-OD groups. Although the tongue strength of the TMD-OD patients was slightly lower than that of TMD patients with no swallowing problems, the difference was not statistically significant. This result could have been due to the inclusion of mixed TMD patients in the study. It can be considered that the tongue muscular strength and endurance in TMD patients may cause different responses to emerge in a muscular, disc, and joint degeneration of the TMJ. The difference in the duration of pain in the two patient groups suggested that it was an important factor affecting tongue strength and endurance. The patients in the TMDOD group had a significantly much longer history of pain. The TMD-OD patients had a mean 3.31-year history of pain, whereas the time from onset of pain to presenting at the clinic for treatment was mean 0.72 years in the TMD patient group without swallowing problems. It can be assumed that there is a relationship between OD and the chronicity of symptoms and pain. From the results of this study, there can be an agreement with the hypothesis that eating and swallowing problems in TMD patients have formed over time secondary to TMD impairments. In a study by Huckabee et al., it was similarly reported that almost half of patients typically experience complaints for 5 years [27].

In the current study, it was seen that in addition to eating and swallowing problems developing secondary to chewing disorders, the maximum mouth opening of the TMD-OD group was significantly decreased compared to the TMD group with no swallowing problems. It was thought that this could be a reason for the longterm chronic pain and increased chewing disorders in the TMD-OD group. In the JFLS-20 evaluation of jaw functional activity, the scores related to chewing, swallowing, and difficulties and restrictions in other jaw movements were found to be higher in the TMD-OD group. In the EAT-10 scale, the symptoms of difficulty and pain in swallowing, the extra effort required for swallowing, adhesion of the bolus to the throat when swallowing, and coughing while eating were experienced more in the TMD-OD group.
There are few studies that have researched the extent and effect of eating and swallowing problems that could accompany TMD. It has been assumed that these difficulties originate at the oral stage and oral preparation of swallowing, that patients do not seek acute medical care for swallowing problems, and that clinicians do not give sufficient importance to these symptoms in the evaluation of TMD [7]. It has also been reported that eating and swallowing problems associated with TMD diminish the quality of life by leading to psychosocial problems [19]. In a previous study, it was reported that in patients with a mean duration of dysfunction of 5 years, there were eating and swallowing problems that could not be effectively managed, and these problems had a moderate negative effect on participation in daily, leisure, social and family activities [7]. Generally, in two-thirds of patients with chronic pain, there are seen to be several accompanying problems such as function loss, withdrawal from social life, fatigue, poor sleep quality, and anxiety [7,27,34]. In the current study, the quality of life of the TMD-OD patients was determined to be lower than that of the other groups. According to the Swal-QoL results, all the categories of food selection, eating time, appetite, fatigue, anxiety, sleep quality, and social and mental health parameters such as social life and communication were seen to be significantly affected in the TMD-OD group compared to the patients without dysphagia.

The aim of this study was to determine clinical differences between patients with TMD and patients who develop OD associated with TMD. The results showed that jaw function, dysphagia symptoms and severity, and quality of life were different in TMD-OD patients compared to patients with TMD without swallowing problems. Moreover, the tongue strength and endurance values of all the TMD patients, with and without OD, were observed to be significantly lower than those of the healthy control group.

In the light of these data, it can be assumed that eating and swallowing problems in TMD patients have developed secondary to chewing disorders. Although the clinical observations and analysis results of this study did not show that this dysfunction caused discomfort to patients at a level to require emergency medical support, it can be considered that over time the quality of life of the individual will be diminished by physical and psychological problems. Therefore, eating and swallowing disorders should be evaluated in patients with chronic TMD in particular, and the inclusion of tongue muscles in treatment programs would be important.

A limitation of this study was that the measurement of oral dysphagia was evaluated subjectively in the clinic environment. A further limitation was that most of the patients were young, and as the patients included 
had mixed TMD diagnoses, detailed categorization could not be made.

In future studies of TMD, the inclusion of a greater number of patients in a wider age range, categorized according to diagnosis criteria, would provide more robust results in the evaluation of tongue strength and endurance in TMD patients with eating and swallowing problems.

\section{Conclusions}

In conclusion, functional restriction of the jaw, swallowing difficulty, and swallowing-related quality of life showed a difference in the patients with TMDOD compared to the patients with TMD without swallowing problems. Patients with TMD were seen to have significantly lower tongue strength and endurance, independently of swallowing problems, compared to the healthy patients. In terms of increasing the success of rehabilitation, the importance must be emphasized of the jaw and swallowing function and evaluation of tongue strength and endurance in the clinical evaluation of TMD patients.

\section{Funding}

This research received no external funding.

\section{Conflict of interests}

The authors have no conflict of interest to declare.

\section{References}

1. Komiyama O, Kawara M, Arai M, Asano T, Kobayashi K. Posture correction as part of behavioural therapy in treatment of myofascial pain with limited opening. J Oral Rehabil. 1999; 26(5): 428-35.

2. Suvinen TI, Reade PC, Kemppainen P, Könönen M, Dworkin SF. Review of aetiological concepts of temporomandibular pain disorders: towards a biopsychosocial model for integration of physical disorder factors with psychological and psychosocial illness impact factors. Eur J Pain. 2005; 9(6): 613-33.

3. Anastassaki Köhler A, Hugoson A, Magnusson T. Prevalence of symptoms indicative of temporomandibular disorders in adults: cross-sectional epidemiological investigations covering two decades. Acta Odontol Scand. 2012; 70(3): 213-23.

4. Logemann, JA. Evaluation And Treatment of Swallowing Disorders. 2nd ed. Austin, Texas:Pro-ed; 1998.

5. Robbins J. Old swallowing and dysphagia: thoughts on intervention and prevention. Nutr Clin Pract. 1999; 14(5 suppl): S21-S26.
6. Ferreira CL, Machado BC, Borges CG, Rodrigues Da Silva MA, Sforza C, De Felício CM. Impaired orofacial motor functions on chronic temporomandibular disorders. J Electromyogr Kinesiol. 2014; 24(4): 56571.

7. Gilheaney Ó, Béchet S, Kerr P, Kenny C, Smith S, Kouider R, et al. The prevalence of oral stage dysphagia in adults presenting with temporomandibular disorders: a systematic review and meta-analysis. Acta Odontol Scand. 2018; 76(6): 448-458.

8. Shaker R. Oropharyngeal Dysphagia. Gastroenterol Hepatol (NY). 2006; 2(9): 633-4.

9. Logemann JA. Swallowing disorders. Best Pract Res Clin Gastroenterol. 2007; 21(4): 563-73.

10. DeMatteo C, Matovich D, Hjartarson A. Comparison of clinical and videofluoroscopic evaluation of children with feeding and swallowing difficulties. Dev Med Child Neurol. 2005; 47(3): 149-57.

11. Plowman EK, Tabor LC, Robison R, Gaziano J, Dion C, Watts SA, et al. Discriminant ability of the Eating Assessment Tool-10 to detect aspiration in individuals with amyotrophic lateral sclerosis. Neurogastroenterol Motil. 2016; 28(1): 85-90.

12. Arrese LC, Schieve HJ, Graham JM, Stephens JA, Carrau RL, Plowman EK. Relationship between oral intake, patient perceived swallowing impairment, and objective videofluoroscopic measures of swallowing in patients with head and neck cancer. Head Neck. 2019; 41(4): 1016-23.

13. Cheney DM, Siddiqui MT, Litts JK, Kuhn MA, Belafsky PC. The Ability of the 10-Item Eating Assessment Tool (EAT-10) to Predict Aspiration Risk in Persons with Dysphagia. Ann Otol Rhinol Laryngol. 2015; 124(5): 351-4.

14. Rosa RR, Bueno MDRS, Migliorucci RR, Brasolotto AG, Genaro KF, Berretin-Felix G. Tongue function and swallowing in individuals with temporomandibular disorders. J Appl Oral Sci. 2020; 28: e20190355.

15. de Felício CM, Medeiros AP, de Oliveira Melchior M. Validity of the 'protocol of oro-facial myofunctional evaluation with scores' for young and adult subjects. J Oral Rehabil. 2012; 39(10): 744-53.

16. Marim GC, Machado BCZ, Trawitzki LVV, de Felício CM. Tongue strength, masticatory and swallowing dysfunction in patients with chronic temporomandibular disorder. Physiol Behav. 2019; 210: 112616.

17. Fassicollo CE, Machado BCZ, Garcia DM, de Felício CM. Swallowing changes related to chronic temporomandibular disorders. Clin Oral Investig. 2019; 23(8): 3287-96.

18. Dworkin SF, LeResche L. Research diagnostic criteria for temporomandibular disorders: review, criteria, examinations and specifications, critique. J Craniomandib Disord. 1992; 6(4): 301-55. 
19. Bijur PE, Silver W, Gallagher EJ. Reliability of the visual analog scale for measurement of acute pain. Acad Emerg Med. 2001; 8(12): 1153-7.

20. Okeson, JP. Management of temporomandibular disorders and occlusion. 3rd ed. Saint Louis; Mosby Year Book; 1998.

21. Shaghayegh Fard B, Ahmadi A, Maroufi N, Sarrafzadeh J. Evaluation of forward head posture in sitting and standing positions. Eur Spine J. 2016; 25(11): 3577-82.

22. Ohrbach R, Larsson P, List T. The jaw functional limitation scale: development, reliability, and validity of 8 -item and 20-item versions. J Orofac Pain. 2008; 22(3): 219-30.

23. Belafsky PC, Mouadeb DA, Rees CJ, Pryor JC, Postma GN, Allen J, et al. Validity and reliability of the Eating Assessment Tool (EAT-10). Ann Otol Rhinol Laryngol. 2008; 117(12): 919-24.

24. Roe JW, Leslie P, Drinnan MJ. Oropharyngeal dysphagia: the experience of patients with non-head and neck cancers receiving specialist palliative care. Palliat Med. 2007; 21(7): 567-74.

25. Solomon NP, Munson B. The effect of jaw position on measures of tongue strength and endurance. J Speech Lang Hear Res. 2004; 47(3): 584-94.

26. Bag AK, Gaddikeri S, Singhal A, Hardin S, Tran BD, Medina JA, et al. Imaging of the temporomandibular joint: An update. World J Radiol. 2014; 6(8): 567-82.

27. Huckabee ML, McIntosh T, Fuller L, Curry M, Thomas P, Walshe M, et al. The Test of Masticating and Swallowing Solids (TOMASS): reliability, validity and international normative data. Int J Lang Commun Disord. 2018; 53(1): 144-56.

28. Youmans SR, Youmans GL, Stierwalt JA. Differences in tongue strength across age and gender: is there a diminished strength reserve? Dysphagia. 2009; 24(1): 57-65.

29. Stierwalt JA, Youmans SR. Tongue measures in individuals with normal and impaired swallowing. Am J Speech Lang Pathol. 2007; 16(2): 148-56.

30. Youmans SR, Stierwalt JA. Measures of tongue function related to normal swallowing. Dysphagia. 2006; 21(2): 102-11.

31. McKenna VS, Zhang B, Haines MB, Kelchner LN. A Systematic Review of Isometric Lingual StrengthTraining Programs in Adults with and without Dysphagia. Am J Speech Lang Pathol. 2017; 26(2): 52439.

32. Takada K, Yashiro K, Sorihashi Y, Morimoto T, Sakuda M. Tongue, jaw, and lip muscle activity and jaw movement during experimental chewing efforts in man. J Dent Res. 1996; 75(8): 1598-606.

33. Valdés C, Astaburuaga F, Falace D, Ramirez V, Manns A. Effect of tongue position on masseter and temporalis electromyographic activity during swallowing and maximal voluntary clenching: a cross-sectional study. J Oral Rehabil. 2014; 41(12): 881-9.

34. Ferreira CLP, Sforza C, Rusconi FME, Castelo PM, Bommarito S. Masticatory behaviour and chewing difficulties in young adults with temporomandibular disorders. J Oral Rehabil. 2019; 46(6): 533-40. 